the wound was closed it was ascertained that only omentum entered the femoral hernia, so this was not interfered with.

The patient's subsequent progress was slow. Though the bowels acted with the help of enemata and flatus passed naturally, yet he was troubled with vomiting and recurring distensions. For several days coils of intestine were mapped out on the abdominal surface. These were first noticed on

Fig: 3.

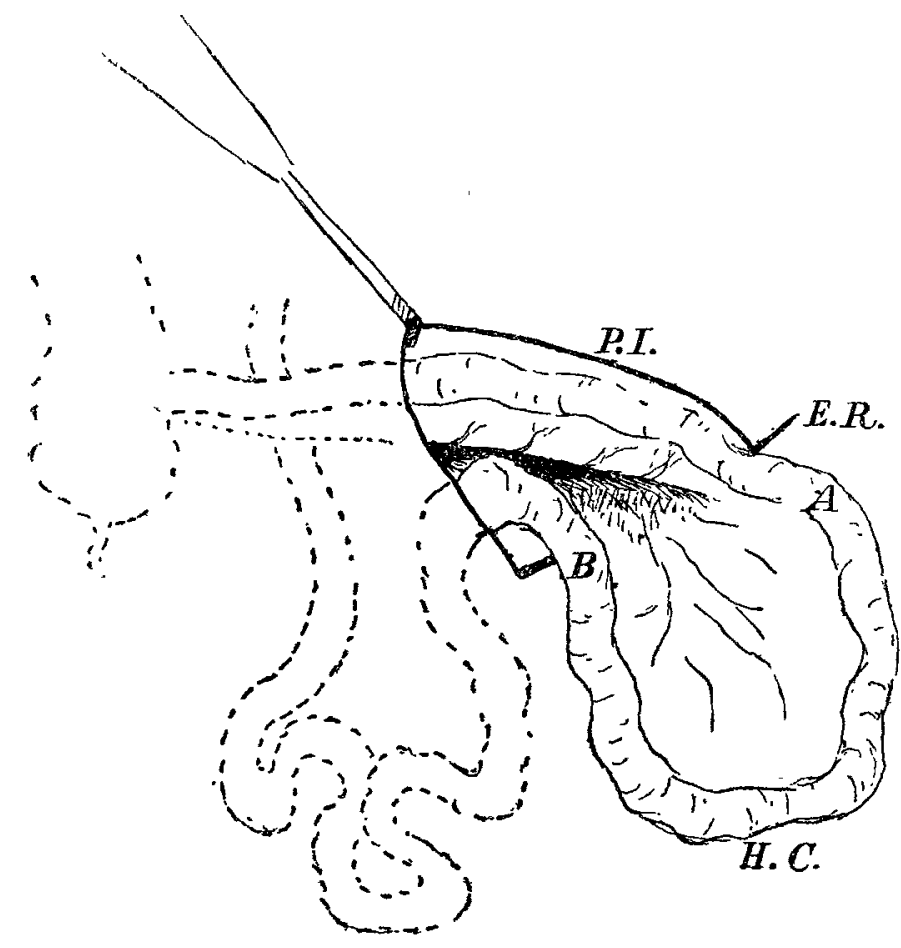

Case 3. Condition disclosed by operation. The dotted lines represent the supposed arrangement of the unseen coils. A, Outer segment of H.C. (herniated coil). B, Inner segment of same. E.R., External ring. P.I., Parietal incision.

Nov. 8th, but a week later he had got over his troubles and was convalescent.

CASE 4. Rught inguinal hernia; torsion of the great omentum. - The patient, a man, aged 31 years, was admitted to the Infirmary 24 hours after the descent of a right inguinal hernia, which he had always controlled with a truss. Pain began as soon as the hernia came down and in two or three minutes it became as large as on admission. He vomited an hour later, but his symptoms were not very acute. The tumour was of the size and shape of a vegetable marrow about eight or nine inches long. Its thick sausage-shaped neck occupied the inguinal canal. The contents of the upper part of the sac were solid and those of the lower were fluid. There. was an impulse on coughing and reduction was impossible.

At the operation, soon after admission, several ounces of bloody fluid and some blood clots escaped from the sac. The solid contents consisted of the great omentum intensely congested and infiltrated with clotted blood. There was no constriction at the neck of the sac and a finger was easily passed into the abdomen. Traction was made upon the omentum and its intra-abdominal portion emerged in a roll having the same engorged appearance as the part that had been present in the sac. The top of the congested roll was reached close to the attachment of the omentum to the transverse colon. The omentum was ligatured in portions and cut away close to the colon and just above the commencement of the engorgement. The case did well.

The explanation of the condition was this. A twist of the great omentum had taken place close to its attachment to the transverse colon, through $180^{\circ}$, from left to right. The left border of the omentum, which was much thickened and rope-like, lay over the right border. When the lower portion of twisted omentum was thrust down into the hernial sac the twist was rendered acute by the traction produced upon its main folds and by the inability of the omentum to alter its position. On examining the specimen the veins were like cords, being greatly distended and filled with clot. Much blood had been effused into the omentum and had collected in firm solid masses as big as Barcelona nuts. There were no adhesions between the twisted parts, and when unrolled the specimen showed a normally shaped great omentum with a crescentic border where it had been cut away from the transverse colon.

Leeds.

\section{A CASE OF VOLVULUS OF THE ENTIRE SMALL INTESTINE, CACUM, AND ASCENDING COLON; OPERA- TION AND RECOVERY.}

By WILLIAM BILlingtoN, M.B., M.S. Lønd., F.R.C.S. ENG.,

SENTOR SURGEON TO OUT-PATIENTS, QUEEN'S HOSPITAL, BIRMTNGHAM,

VERY few instances of this form of volvulus are on record. Mr. Jonathan Hutchinson, jun., in a paper which appeared in the Clinucal Journal of June 5th, 1907, was only able to collect ten cases, and of these only two recovered. It can only occur when the attachment of the ascending colon and cæcum to the posterior abdominal wall is extremely loosei.e., in a floating condition. This is undoubtedly of congenital origin, and most of the cases of complete volvulus have been met with in subjects under 25 years of age. In all the cases recorded and in the one about to be described the third stage of the duodenum was also freely moveable and indistinguishable from the second stage.

In this form of volvulus the whole of the small intestine, the cxcum, and the ascending colon are rotated axially, the cæcum being carried behind the mesentery into the left side of the abdomen, being found in my case at the level of the spleen. The small intestine comes to lie to the right of the cæcum and some of the coils, becoming heavy with fluid, gravitate into the pelvis, tightening their mesentery, which crosses the displaced colon. The mesentery thus becomes a tight band which compresses the colon and effectually prevents the contents of the cæcum from passing on. This causes great distension, especially of the cæcum, since the small intestine can pour its contents into the cæcum as into a cistern from which there is no outlet.

A man, aged 41 years, came to see me on Oct. 1st, 1908. He was perfectly well until eight or nine years ago, when he noticed blood in his urine and passed a small calculus per urethram. About 12 months later he began to have frequent attacks of renal colic and passed numerous small stones by the urethra. At this time he was cystoscoped with a negative result. Three years ago, when on a visit to London, he was seized with a severe attack of renal colic and attended St. Peter's Hospital where he was examined by Mr. P.J. Freyer. Pus was seen escaping from the right ureter, but as an $x$ ray examination failed to show the presence of a stone nothing was done. For the last two years there had been pus in every specimen of urine and he had had an attack of severe colic at intervals of eight or nine months, each attack being followed by the passage of a stone by the urethra. The last stone was passed at Christmas, 1907. For a fortnight prior to my seeing him the urine had been quite clear, but he had suffered from headache, pains in the limbs, and profuse sweating, and he had wasted. On the morning of his visit he had passed about a pint of what looked like pure pus per urethram. On four or five occasions during the last four years, at intervals of eight or nine months, there had been complete stoppage of the bowels, lasting for about two days. At other times the bowels were regular and gave no trouble. As these attacks were coincident with the renal colic no particular attention was directed to them.

The patient was a tall, thin man who looked haggard and emaciated. He brought with him a quantity of thick pus of acid reaction which he said he had passed that morning. The right kidney was very moveable and enlarged but not tender. The left kidney came down two or three inches below the costal margin and did not appear to be enlarged. Examination of the chest and abdomen revealed nothing of interest. As there were no indications for immediate interference and the patient was weak and ill he was advised to rest quietly at home for a month and then to present himself for a more detailed examination into the state of his kidneys, with a view to operation. On 
account of the mobility of the kidneys an abdominal belt giving special support to the kidneys by means of rubber airpads was ordered. This belt was probably instrumental in causing the subsequent attack of intestinal obstruction, since the right air-pad would tend to displace the cxcum to the left.

For some time the patient went on very well and his general condition improved considerably. Further, he expressed himself as having derived considerable comfort from the belt. Rather more than three weeks after his visit to me, he was seized with colicky abdominal pain and complete stoppage of the bowels. As this had occurred before, no great notice was taken of it. The pain, however, got worse, and two days later he began to vomit. A medical man was called in on the fourth day of the attack and various aperients and numerous enemata were tried with negative result. The pain was now so severe that the patient could not lie down but spent his time sitting in a chair and walking about. I was asked to see him on Nov. 5th, six days after the onset of the attack. He was sitting on the edge of the bed and looked very ill, the face being drawn and pinched. After taking a mixture containing opium the previous day the vomiting had entirely ceased and the pain was much easier, but at intervals he still had severe spasms of colic. The tongue was brown but moist, the pulse was 110 , but strong and good, and the temperature was $98 \cdot 4 \circ \mathrm{F}$. Nothing whatever had been passed by the rectum for six days. The abdomen was moderately distended all over and on the left side, filling up the entire space between the costal margin and a horizontal line at the level of the umbilicus, was a tense rounded swelling which stood up strikingly above the general level of the abdomen. It was tympanitic on percussion and could not be moved. The maximum projection was several inches below the costal margin but the fingers could not be passed between the costal margin and the tumour. As it was late at night and the surroundings were unsuited for an abdominal section in the patient's own home, high turpentine enemata were ordered and instructions were left that if not better on the following day be should be moved into a nursing home for immediate operation. Delay seemed justified by the fact that both the patient and his medical adviser were satisfied that his general condition had improved since the preceding day. On the next day, as the obstruction remained unrelieved and the vomiting had returned, he was transferred to a nursing home and operation was carried out. The vomiting occurred at short intervals but only a small quantity of greenish fluid with a slight fæculent odour was brought up each time. The pulse-rate had increased to 126 .

Operation.-An incision was made through the right rectus muscle for the reason that it seemed possible that the enlarged right kidney might in some way have induced the obstruction. On opening the peritoneum a quantity of slightly blood-stained fluid escaped. Many distended coils of small intestine were found beneath the incision and on opening these out the mesentery was seen to be twisted and greatly stretched. It was much congested and showed many petechial hrmorrhages. The tumour in the left side was found to consist of the enormously distended cæcum and first part of the ascending colon. Just to the right of the middle line the ascending colon was crossed by the tightly stretched twisted mesentery which completely occluded the bowel. The cæcum was punctured when a great volume of gas and 60 ounces of liquid frees escaped. It was then found possible to lift the small intestines over to the left side of the abdomen and to bring the cæcum from behind the mesentery. While doing this the lumen of the small bowel near the duodenum was found occluded by a twist round the longitudinal axis of the bowel. This accounted for the fact that the vomit had been small in amount and practically non-fæculent. After operation a considerable quantity of brown fæculent matter was vomited. The third stage of the duodenum was freely moveable and directly continuous with the second stage. Finally, the abdominal wound was sewn up without drainage. To prevent a recurrence of the volvulus and to allow of an opening being easily made into the bowel in case of necessity a pouch of the huge flaccid creum was fixed by sutures to the anterior sheath of the rectus in the lower angle of the wound.

The patient stood the operation well. He vomited several times during the first 24 hours, the vomit consisting at first of strongly fæculent brown fluid and later of bile. Flatus was passed by the rectum after a few hours and the bowels acted naturally at the end of 48 hours. The pouch of cæcum was not opened and soon healed over, though some weakness of the scar and slight bulging on coughing persisted at that point. An uneventful recovery took place and at the end of a month he was able to return home feeling quite strong and better than he had been for two years. The urine still contained a moderate amount of pus and in all probability the right kidney will have to be explored in the near future.

This case is recorded on account of the rarity of the condition and the many interesting features it presented.

Birmingham.

\section{TWO CASES OF VOLVULUS ASSOCIATED WITH HERNIA.}

BY J. F. DOBSON, M.S. LOND., F.R.C.S. ENG., CLINICAT, LECTURER ON SURGERT, UNIVERSTTY OF LEEDS, ETC.

CASE 1.-A man, aged 43 years, was admitted to the Leeds General Infirmary on July 20th, 1907. He had suffered from a right inguinal hernia for some years which had not previously given him any trouble. 48 hours before admission he was seized with pain in the hernia, which became swollen and irreducible, and in the abdomen. The bowels had not been moved and vomiting had been constant. On admission he was in a state of collapse, with cold extremities and a rapid feeble pulse. There was a large, tender, irreducible inguinal hernia on the right side, which gave no impulse on coughing. The abdomen was distended and tender, but it was possible to make out a tense swelling in the right iliac region extending upwards towards the umbilicus of about the size of the foetal head. A diagnosis was made of a volvulus of the small intestine lying partly in the abdomen and partly in the hernial sac.

Under stovaine spinal anæsthesia an incision was made in the middle line below the umbilicus. There was a large quantity of blood-stained fluid, having a fæcal odour, in the abdominal cavity. Some 7 or 8 feet of the lower ileum were found to be twisted on its mesenteric attachment and lying partly in the hernial sac. The gut was of a deep plum colour with dark patches in places. The whole of the volvulus was excised and owing to the desperate condition of the patient Paul's tubes were inserted into the two ends ; the tubes were surrounded with ganze and the abdomen was closed as rapidly as possible. Death occurred 36 hours later.

CAsE 2-A man, aged 68 years, was admitted to the infirmary on June 13th, 1908. He had suffered from a right inguinal hernia for many years. On June 12th the hernia became suddenly painful and swollen and could not be reduced. This was followed by vomiting which continued up to the time of his admission.

The case was thought to be an ordinary strangulated hernia and, under chloroform, herniotomy was performed in the usual way. On opening the sac, which contained a quantity of fluid, coils of normal small intestine were found lying side by side with other coils which were distended, thickened, and congested. There was no constriction at the neck of the sac, and on tracing the distended loops of gut upwards they were found to be continuous with other similar loops within the abdomen; these were withdrawn and it was then found that there was a volvulus of the small intestine involving two or three feet, the gut being twisted through one complete turn. The volvulus was untwisted and the intestine was returned into the abdomen. There was a large chronic vaginal hydrocele on the same side, and permission having been previously obtained the testicle was removed and the inguinal canal completely closed. Recovery ensued without incident.

The first of these two cases is of great interest in that it was possible to recognise the condition before operation and so to make an abdominal incision instead of one over the hernia, which would have been quite inadequate. It so happened that a short time previously I had re-read a reprint of Mr. R. Lawford Knaggs's paper in the Annals of Surgery for April, 1900, and recognition of the condition was therefore an easy matter. Both cases were of the same type, part of the volvulus lying in the hernial sac and part in the abdomen; in the first case many feet (probably about 8 ) of intestine were affected, while in the second case the volvulus 